\title{
Delta Variant: Expanding the Indication for Eluxadoline to Bile Acid Diarrhea
}

\author{
Eric D. Shah ${ }^{1}$
}

Accepted: 3 January 2022 / Published online: 5 February 2022

(c) The Author(s), under exclusive licence to Springer Science+Business Media, LLC, part of Springer Nature 2022

Diarrhea-predominant irritable bowel syndrome (IBS-D) is a heterogeneous condition that is broadly defined by the presence of chronic abdominal pain and loose stools [1]. As carefully delineated in a recent narrative review by Camilleri et al., bile acid diarrhea (BAD) is a disease characterized by enteric malabsorption of bile acids that are important to enterohepatic communication and gut microbiota signaling [2]. Indeed, the primary symptoms of BAD include urgency to defecate, abdominal pain, and occasional accidental bowel leakage/fecal incontinence. The management paradigm for BAD has focused sensibly on identifying bile acid malabsorption and to broadly provide bile acid sequestrants or to promote a low-fat diet as the field has emerged over the past decades. More recently, ongoing clinical trials have demonstrated that investigational nuclear farnesoid $\mathrm{X}$ receptor (FXR) agonists are capable of improving key symptoms of BAD in a manner that more specifically targets the underlying pathophysiologic mechanism by decreasing the synthesis of hepatic bile acids through feedback inhibition [3]. Given that symptoms of IBS-D and BAD significantly overlap, it is possible that patients with BAD may be empirically treated with non-targeted IBS-D treatments and that patients with BAD might experience some level of general clinical improvement with this purely symptom-based approach to care [4]. To truly achieve the promise of personalized medicine, there remains a clear opportunity for diagnostic paradigms to more accurately target therapy tied to underlying disease mechanisms such as BAD.

Eluxadoline is an FDA-approved as a safe and effective treatment for IBS-D [5]. Eluxadoline is a peripheral mixed $\mu$ - and $\kappa$-opioid receptor agonist and $\delta$-opioid receptor

Eric D. Shah

eric.d.shah@hitchcock.org

1 Center for Gastrointestinal Motility, Esophageal, and Swallowing Disorders, Division of Gastroenterology and Hepatology, Dartmouth-Hitchcock Medical Center, One Medical Center Drive, Lebanon, NH 03766, USA antagonist that reduces visceral hyperalgesia while minimizing the risks of slowing motility beyond a therapeutic range. The latter behavior of eluxadoline is proposed to be due to its $\delta$-opioid receptor antagonism that mitigates the constipating effects of pure $\mu$-opioid receptor agonists such as loperamide while conferring analgesic properties [6]. Though eluxadoline is approved for IBS-D and not for BAD, patients with BAD have similar symptoms as those with IBS-D. Therefore, the wide-ranging anti-diarrheal property of eluxadoline lends itself to the possibility that symptoms of BAD might improve with treatment.

In this issue of Digestive Diseases and Sciences, Vijayvargiya et al. [7] from the Mayo Clinic performed a rigorous, open-label cohort study on patients meeting Rome IV criteria for IBS-D who received eluxadoline at its FDAapproved dose of $100 \mathrm{mg}$ twice daily for 4 weeks (including an appropriate 2-week run-in period). The purpose of the study was to distinguish pathophysiologic entities such as BAD from all-encompassing symptom-based classifications such as IBS-D. All patients underwent baseline diagnostics for bile acid diarrhea using fasting serum $7 \alpha$-hydroxy-4cholesten-3-one ( $7 \alpha-C 4)$ levels, a biomarker of hepatic bile acid synthesis; this test has been previously validated to have a sensitivity of $90 \%$ and specificity of $79 \%$ to detect bile acid malabsorption [8]. Ultimately, the investigators found that eluxadoline mostly improved symptoms of IBS-D regardless of the presence of BAD.

This study is particularly important to the field of several reasons. First, there remains a core need to support equal research and development efforts on both diagnostics as has been done in $\mathrm{BAD}$, considering the development of $7 \alpha-\mathrm{C} 4$ testing as well as 48 -h fecal bile acid excretion or ${ }^{75} \mathrm{Se}$ homocholic acid taurine (SeHCAT) retention as well as proposed treatments such as investigational FXR agonists that target specific and unified mechanisms. Regardless, the general anti-diarrheal properties of eluxadoline lend its similar efficacy among patients with IBS-D regardless of the presence of BAD. Second, many clinicians use empiric 
bile acid sequestrants in daily practice $[9,10]$. As outlined in recent clinical practice guidelines, it is important to recognize that a positive outcome with bile acid sequestrants does not necessarily indicate the presence of BAD_-just as the Rome IV criteria do not support a positive diagnosis of IBS-D based on a trial of eluxadoline [11]. Given the relatively non-targeted effects of bile acid sequestrants as noted by Camilleri et al., there remains a need to develop safe, effective, and specific agents that more effectively target the underlying mechanisms of BAD as is presently underway. Finally, diagnostic testing is important not only to identify appropriate treatment avenues but also serves to validate the patient experience, especially for chronic illnesses that significantly impair quality-of-life $[9,10]$.

In summary, the work of these authors and the many others that strive to define personalized care paradigms that will gain further importance in the treatment of patients affected by disorders of gut-brain interaction, gastrointestinal motility disorders, and related diseases that share overlapping symptoms in the coming decade.

Funding Eric Shah is supported by the AGA Research Foundation's 2019 AGA-Shire Research Scholar Award in Functional GI and Motility Disorders.

\section{Declarations}

Conflict of interest EDS consulted for Bausch Health and GI Supply outside the scope of the current work.

\section{References}

1. Lacy BE, Pimentel M, Brenner DM, Chey WD, Keefer LA, Long $\mathrm{MD}$ et al. ACG clinical guideline: management of irritable bowel syndrome. Am J Gastroenterol 2021;116:17-44.
2. Camilleri $\mathrm{M}$, Vijayvargiya $\mathrm{P}$. The role of bile acids in chronic diarrhea. Am J Gastroenterol 2020;115:1596-1603.

3. Camilleri M, Nord SL, Burton D, Oduyebo I, Zhang Y, Chen $\mathrm{J}$ et al. Randomised clinical trial: significant biochemical and colonic transit effects of the farnesoid $\mathrm{X}$ receptor agonist tropifexor in patients with primary bile acid diarrhoea. Aliment Pharmacol Ther 2020;52:808-820.

4. Shah ED, Chang L, Salwen-Deremer JK, Gibson PR, Keefer L, Muir JG et al. Contrasting clinician and insurer perspectives to managing irritable bowel syndrome: multilevel modeling analysis. Am J Gastroenterol 2020;116:748. https://doi.org/10.14309/ajg. 0000000000000989.

5. Lembo AJ, Lacy BE, Zuckerman MJ, Schey R, Dove LS, Andrae DA et al. Eluxadoline for irritable bowel syndrome with diarrhea. N Engl J Med 2016;374:242-253.

6. Fujita W, Gomes I, Dove LS, Prohaska D, McIntyre G, Devi LA. Molecular characterization of eluxadoline as a potential ligand targeting mu-delta opioid receptor heteromers. Biochem Pharmacol 2014;92:448-456.

7. Vijayvargiya et al. Safety and efficacy of eluxadoline in patients with irritable bowel syndrome-diarrhea with or without bile acid diarrhea: open-label study. Dig Dis Sci. (Epub ahead of print). https://doi.org/10.1007/s10620-022-07379-x.

8. Sauter GH, Münzing W, von Ritter C, Paumgartner G. Bile acid malabsorption as a cause of chronic diarrhea: diagnostic value of 7alpha-hydroxy-4-cholesten-3-one in serum. Dig Dis Sci. 1999;44:14-19.

9. Sadowski DC, Camilleri M, Chey WD, Leontiadis GI, Marshall $\mathrm{JK}$, Shaffer EA et al. Canadian association of gastroenterology clinical practice guideline on the management of bile acid diarrhea. Clin Gastroenterol Hepatol 2020;18:24-41.e1.

10. Smalley W, Falck-Ytter C, Carrasco Labra A. AGA clinical practice guidelines on the laboratory evaluation of functional diarrhea and diarrhea-predominant irritable bowel syndrome in adults (IBS-D): patient summary. Gastroenterology 2019;157:856-857.

11. Lacy BE, Mearin F, Chang L, Chey WD, Lembo AJ, Simren M et al. Bowel disorders. Gastroenterology. 2016;150:1393-1407.e5.

Publisher's Note Springer Nature remains neutral with regard to jurisdictional claims in published maps and institutional affiliations. 